\title{
Design and implementation of the hand venipuncture auxiliary system based on cloud computing platform
}

\author{
Jiaying Dang * \\ School of Information Engineering, Communication Engineering, Wuhan University of Technology, Wuhan \\ City, Hubei Province, 430000 \\ China \\ * Corresponding author email: 1793469410@qq.com
}

Keywords: Cloud computing platform; Venous image processing; Multi-mode projection of intravenous lines; The database of matching identity information with intravenous lines; Auxiliary puncture.

\begin{abstract}
In clinical medicine, venipuncture has become a highly used medical operation. However, with the improvement of people's living standards, the number of obese users is increasing, and the number of infants, the elderly and ICU patients is increasing, so that the rate of difficulty in piercing is increasing. In order to solve the problem of low accuracy of venipuncture technology, this paper proposes a design of a hand venipuncture auxiliary system based on cloud computing platform. The system consists of venous projection terminal, cloud computing platform and APP. The infrared image acquisition module of the terminal of vein projection can collect vein image. The wi-fi module can realize the function of information exchange with the cloud computing platform. The projection module can realize multi-mode projection of vein. By running RM vein image processing and multi-function projection algorithm, as well as the matching database system of intravenous lines and identity information, the cloud platform can control the switch of projection mode in real time and transmit the user's medical treatment results to the APP in real time. The APP performs the storage of users' veins, displays the medical information in real time, and provides users with healthy health suggestions according to the physical health problems displayed by the user's medical information. This design adopts the combination of vein image processing and functional projection algorithm based on cloud platform and matching database to realize an intelligent and efficient hand venipuncture auxiliary system. Experimental results show that the system can effectively improve the accuracy of puncture.
\end{abstract}

\section{Introduction}

In today's society, with the improvement of people's living standards, the number of obese users is increasing, while the number of people with puncture difficulties is also increasing, making the probability of puncture difficulties increasing. Therefore, there is a growing demand for intelligent and efficient auxiliary equipment for venipuncture. At present, there is a lot of auxiliary equipment for venipuncture in the market, but they all have the following drawbacks: (1) most of the auxiliary equipment for venipuncture displays vein lines on a screen, but the screen monitoring area is separated from the doctor's operating area, which changes the operation habit of medical staff and reduces the efficiency of medical staff.(2) some auxiliary equipment for venipuncture requires professional personnel to control the equipment on site, the intelligence degree of which is also very low.(3) some products can only provide projection of intravenous lines in a single mode, which limits the environment in which the device is used and reduces the probability of successful puncture. In view of the above situation, in order to improve the probability of successful puncture more intelligently and efficiently, this paper proposes a hand venipuncture auxiliary system based on cloud computing platform. This paper first describes the overall design of the system, and describes the functions and implementation of the system from three parts: the venous projection terminal, the 
cloud platform and the user APP. Then it introduces the core algorithms, including vein image processing and multi-function projection algorithm, and the system of matching the user vein pattern and identity information to the database. Secondly, this paper describes the overall working process system of the system and analyzes the experimental results. Finally, the conclusion is drawn and the whole paper is summarized [1][2].

\section{Overall system design}

As shown in Figure 1, the system consists of venous projection terminal, cloud platform and APP. The infrared image acquisition module of the terminal of vein projection can collect vein image. The wi-fi module can realize the function of information exchange with the cloud computing platform. The projection module can realize multi-mode projection of vein. By running RM vein image processing and multi-function projection algorithm, as well as the matching database system of intravenous lines and identity information, the cloud computing platform can control the switch of projection mode in real time and transmit the user's medical treatment results to the APP in real time. The APP performs the storage of users' intravenous lines, displays the medical information in real time, and provides users with healthy health Suggestions according to their physical health problems.

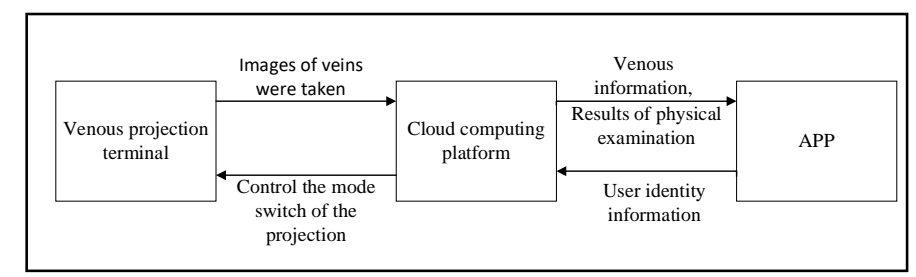

Fig.1 System Overall Design Diagram

\subsection{Venous projection terminal design}

The venous vascular projection terminal is based on the STM32 processor, which also includes the infrared image acquisition module and wi-fi module of the venous vessels, and the DLP projection module that projects the processed venous vascular images received by the wi-fi receiving module onto the surface of the user's puncture site. Finally, it realizes direct interaction with the user in the form of projection. In this system, the LRCP10190_1080P infrared camera is used to collect the infrared image of the vein of the user to be pierced, and the infrared image of the vein collected is transmitted to the cloud computing platform in the form of MJPG through the wi-fi module. This system uses the DLP projection device to project the processed venous image received by the wi-fi module onto the surface of the parts to be punctured. The projection module also adopts the optical path combination of the projected optical axis and the shooting optical axis, which is orthogonal to each other, through the $45^{\circ}$ infrared filter, and adopts the deviation adjustment to make the projected image effectively coincide with the real vein image, so as to achieve accurate registration of different planes. As shown in figure 2, it is the systematic framework of the projection part of the vein. The wi-fi wireless transmission part of this design adopts the LHW100A module. The wireless transmission part is embedded with the TCP/IP protocol, which can carry out real-time communication with the network server, realize the interconnection with the cloud computing platform, and carry out real-time exchange of information. 


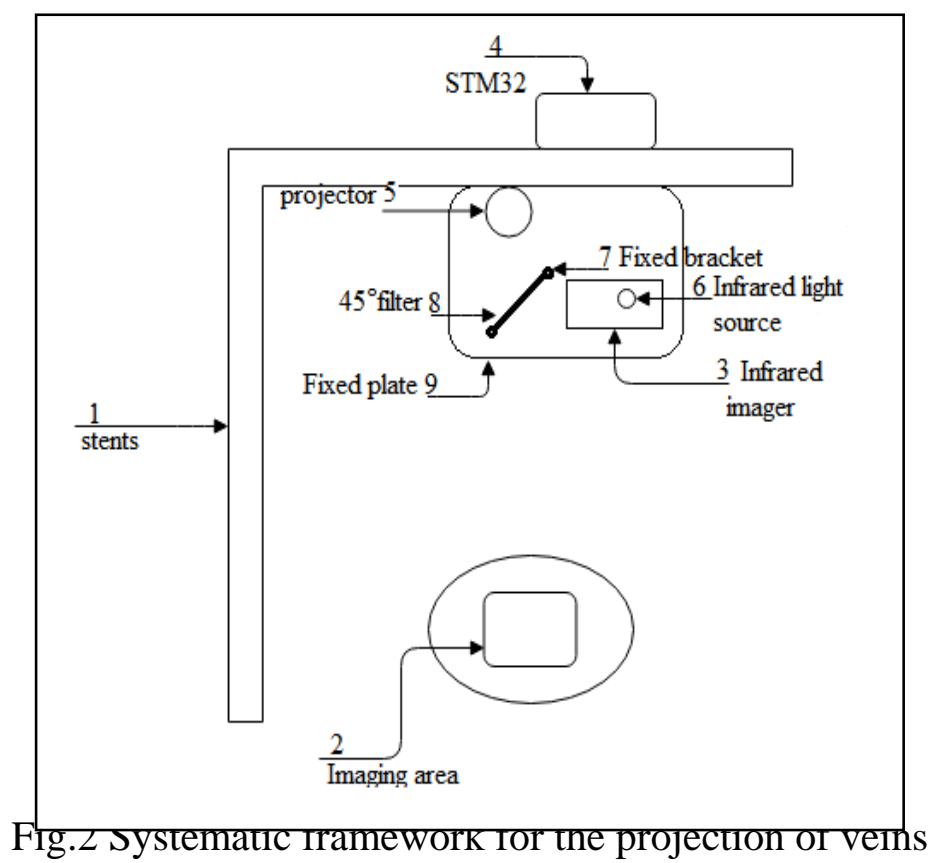

\subsection{Cloud computing platform design}

The main function of a cloud computing platform is to execute algorithms. By executing RM venous image processing and multi-function projection algorithm, the cloud computing platform processes the infrared image of the vein sent by the wi-fi module and transmits it to the wi-fi receiving module in the form of projection. At the same time, the cloud computing platform can also match the identity of the user's medical information with the intravenous lines by the database system for matching intravenous lines with identity information, and transmit the user's medical treatment results to the APP in real time.

\subsection{User APP design}

App is the interactive interface between the system and the user, which can realize the function of inputting the user's characteristic information. The design of the APP can display user's intravenous lines, and through matching the registered user id number, gender, age and other personal information with intravenous lines one by one, the user can view the medical treatment result in the APP. The APP can also provides users with healthy health-preserving Suggestions according to their physical health problems shown by the user's medical information.

\section{Multi-function projection of vein and identity information matching of intravenous lines}

The core algorithms of this system include RM vein image processing and multi-function projection algorithm and the database system algorithm for matching intravenous lines and identity information. The former is mainly used to process infrared images of veins and control the switch of projection mode in real time; the latter is used to identify and match the user's medical information with the user's intravenous lines, and the latter can also transmit the user's medical treatment result to APP in real time.

\subsection{Venous image processing and multi-function projection algorithm}

This system adopts RM vein image processing and multi-function projection algorithm to process the infrared image of intravenous lines and realize the projection imaging of different modes. In this system, the venous image processing algorithm is mainly composed of three structural modules: filtering and denoising, morphological processing and optimal depth search. Different algorithms are adopted according to the needs of each structural module to ensure accurate processing and ecological repair of vascular images collected under different conditions. The multi-function 
projection mode switching algorithm is mainly composed of four structural modules: color switching, brightness adjustment, inversion display and depth recognition, realizing real-time switching of projection mode according to user requirements.

In this system, Frangi algorithm is used for edge detection and enhanced filtering[3], niBlackThreshold() algorithm is binarization processing, dilate algorithm is morphological processing, and three-layer depth extraction algorithm is depth search. The block diagram of the venous image processing system of this system is shown in figure 3.

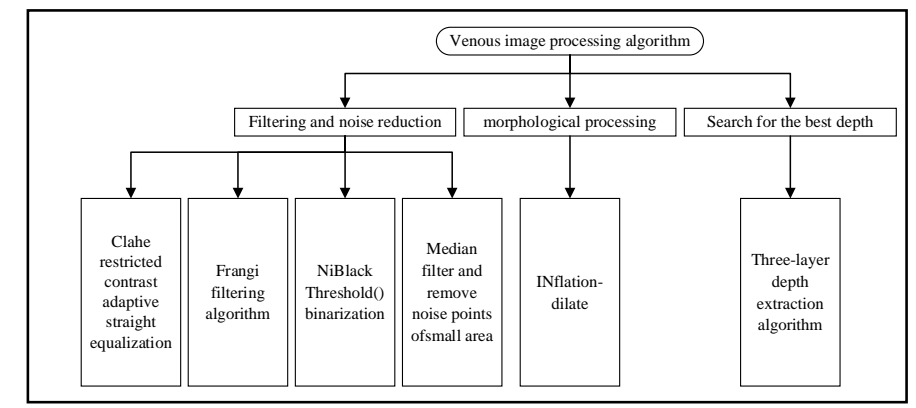

Fig.3 Block diagram of venous image processing system

The multi-function projection algorithm of intravenous lines in this system can realize the adjustment of projection color by using cv Color algorithm. Medical staff can adjust the projection color according to their own optimal fitness of color to achieve the best puncture effect. The contrast Change algorithm can adjust the projection brightness, and the medical staff can choose different projection brightness according to their sensitivity to the projection brightness, so as to ensure the probability of successful puncture. This system can use the depth Detect algorithm to extract the depth and find the best puncture site of the user. The reversal algorithm can reverse the background and increase the contrast so that the vascular image can be projected more clearly. Scale Transformation algorithm can realize the transformation between the projection mode of children's local fine image and the full-projection mode of adult image, so as to adapt to different puncture groups and improve puncture accuracy.

\subsection{The system for matching user vein pattern and identity information}

Through the database system, the system matches the intravenous lines of the user's hand with the id card number, gender, age and other personal information filled in by the user during APP registration and transmits the user's medical treatment results to the database in real time. The APP can display the medical information in real time by extracting the database location of the user and provide users with healthy health-preserving suggestions according to their physical health problems shown by the user's medical information.

\section{System workflow}

The working process of the system is as follows: the terminal is connected to the cloud computing platform through ESP8266, and use can uploads the user's id number, gender, age and other personal information in the APP. During work, the venous image collection part collects the user's hand vein image and uploads it to the cloud computing platform. The cloud computing platform controls the switch of projection mode in real time by running RM vein image processing and multi-function projection algorithm, as well as the database system of matching intravenous lines with identity information. And it can also transmit the user's medical treatment results to the APP in real time. The APP performs the storage of the user's intravenous lines, displays the medical information in real time, and provides users with healthy health-preserving suggestions according to their physical health problems shown by the user's medical information. 


\section{Results and analysis}

\subsection{System prototype design}

The model of the system was built with Rhino software, and the prototype design of the system was carried out as shown in figure 4. In figure 4, the system prototype is expanded and folded from left to right.

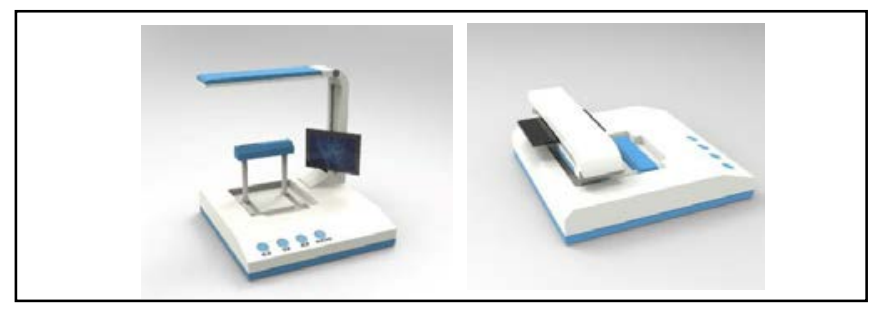

Fig.4 System prototype design

\subsection{Projection results of multi-function mode}

The projection results of this system are divided into four modes: color switching, brightness adjustment, inversion display and depth recognition. As shown in figure 5, the projected colors can be switched between red, green, and purple.In figure 6, from left to right, the results of the weaker mode and the brighter mode are shown.As shown in figure 7, the projection background conversion displays.As shown in figure 8, the optimal puncture location for projection depth recognition is calibrated.

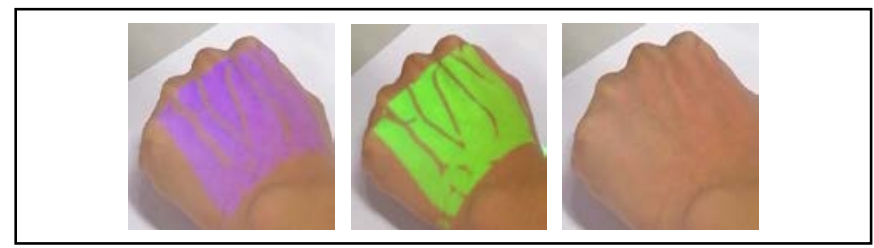

Fig.5 Red, green and purple three projection color conversion

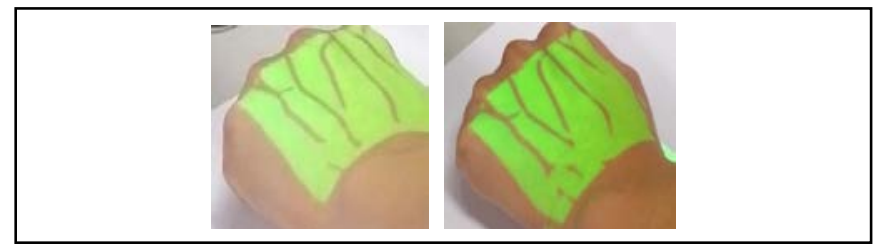

Fig.6 Brightness adjustment of projection

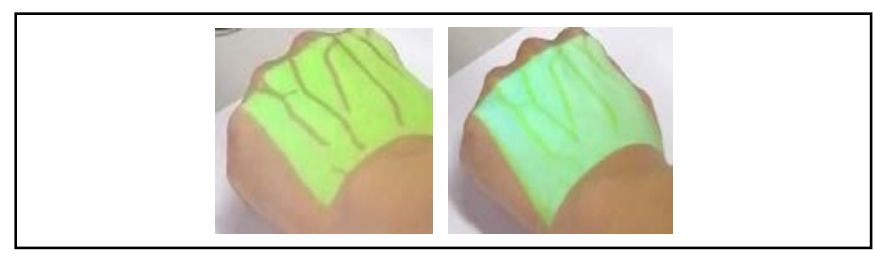

Fig.7 Background transformation of projection of veins in hands

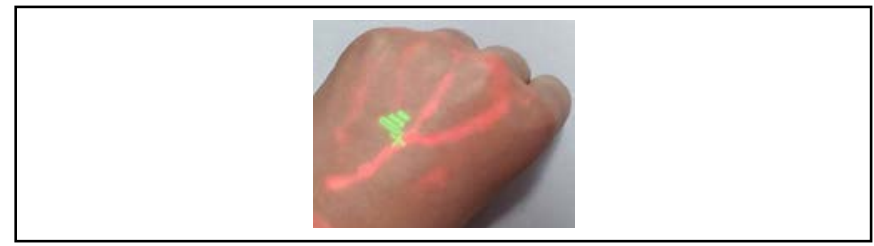

Fig.8 Calibration of the best puncture location of the hand vein

\subsection{Analysis of puncture success rate}


Under the condition of control variables, 128 cases were set as experimental group and 128 cases as control group in the test. The experimental group adopted the auxiliary equipment of hand venipuncture, while the control group adopted the traditional puncture method, respectively (1) one-time success rate of venipuncture: the patient is punctured by medical staff. If the infusion is unobstructed, without extravasation, swelling and other adverse reactions after 3 to 5 minutes, the puncture is successful[4].(2) time to establish a single venous access: that is, the time from the ligation of the tourniquet to the end of the puncture.(3) incidence of pain: in this study, the wong-baker facial expression scale was used to evaluate the pain, with $0 \sim 2$ as painless and $3 \sim 5$ as pain[5].The test results are shown in table 1.

Table 1Experimental test results.

\begin{tabular}{|c|c|c|c|c|}
\hline group & $\begin{array}{c}\text { The number } \\
\text { of cases }\end{array}$ & $\begin{array}{c}\text { Success of one-time } \\
\text { venipuncture } \\
\text { [case (\%)] }\end{array}$ & $\begin{array}{c}\text { Time consuming to establish } \\
\text { a single venous access } \\
(\mathrm{s}, \bar{x} \pm s\end{array}$ & $\begin{array}{c}\text { The pain } \\
\text { [case } \\
\text { (\%)] }\end{array}$ \\
\hline control group & 128 & $110(85.93)$ & $89.56 \pm 7.92$ & $25(19.53)$ \\
\hline $\begin{array}{c}\text { experimental } \\
\text { group }\end{array}$ & 128 & $124(96.88)$ & $61.24 \pm 8.03$ & $4(3.13)$ \\
\hline
\end{tabular}

Experimental results showed that the success rate of one-way venipuncture in the experimental group was $96.88 \%$, significantly higher than the success rate of $85.93 \%$ in the control group; the time consuming to establish a single venous access in the experimental group was 61.24s, less than 89.56s in the control group, and the pain incidence was also significantly lower than that in the control group.To sum up, the auxiliary device of hand venipuncture based on cloud computing platform in this system can significantly improve the success rate of puncture and reduce the pain of patients.

\section{Conclusion}

The hand venipuncture auxiliary system based on cloud computing platform is mainly composed of three parts: venous projection terminal, cloud computing platform and APP. Venous projection terminal can realize venous image acquisition, information interaction with cloud computing platform and projection function. The cloud computing platform controls the switch of projection mode in real time by running RM vein image processing and multi-function projection algorithm, as well as the database system of matching intravenous lines with identity information. And it can also transmit the user's medical treatment results to the APP in real time.The APP performs the storage of the user's intravenous lines, displays the medical information in real time, and provides users with healthy health-preserving suggestions according to their physical health problems shown by the user's medical information. However, in the context of more complex user information and external conditions, the system can't more accurately analyze the image of veins, so we hope to design more complex and comprehensive algorithms to improve the system in the future.

\section{References}

[1] Sullivan M.Improving patient safety with intelligent infusion devices[J].AmJHealthSystPharm, 2010, 67(17):14-15.

[2] Lirong Zhang, Shasha liu . Research progress on safety management of intravenous infusion in China [J]. Nursing research, 2013,2 (3C) : 774-776. (in Chinese)

[3] JERMAN T, PERNUS F,LIKAR B,et al.Beyond Frangi:an improved multiscale vesselness filter[C]/Proc.SPIE 9413,Medical Imaging 2015:Image Processing,94132A (2015).

[4] Xuejin Zhao. Method and effect analysis of improving the success rate of venipuncture [J]. Modern clinical nursing, 2005,4 (1) : 63-65. 
[5] Ke Tang. Measurement and evaluation of patient pain [J]. Contemporary nurses: academic edition, 2008,15 (9): 7-9. 